\title{
The relationship between vascularity, p53 gene mutations and distant metastatic disease in epithelial ovarian carcinoma
}

\author{
Janna Girardi, ${ }^{1}$ Megan Samuelson, ${ }^{2}$ Anna Button, ${ }^{3}$ Koen DeGeest, ${ }^{1,4}$ David Bender, ${ }^{1,4}$
} Amina Ahmed, ${ }^{1,4}$ Michael J Goodheart ${ }^{1,4}$

\section{Objective:}

Angiogenesis is a critical component in the development of metastatic disease in patients with epithelial ovarian carcinoma. Mutations of the p53 gene are frequent in epithelial ovarian cancer and have been associated with an increase in angiogenesis. We hypothesize changes in tumor vascularity are associated with mutations in the p53 gene in patients with metastatic epithelial ovarian carcinoma.

\section{Methods:}

Searching our divisional database, we identified patients diagnosed with stage IIIC or IV epithelial ovarian carcinoma who had undergone primary surgery and had tissue available for analysis from both primary and metastatic disease sites. Microscopic slides were cut from paraffin-embedded tissue and stained for the vascular endothelial marker CD31. Microvessel density (MVD) counts were determined by averaging three high power fields within the tumor section with the greatest concentration of tumor and were expressed as vessels/high power field (HPF). The average MVD counts for metastatic sites were determined under microscopic examination and compared to MVD counts from primary ovarian cancer sites. Clinical and pathologic factors included in the analysis were histology, grade, and p53 mutational status. Absolute differences in MVD counts between primary and distant tissue sites of $>7$ vessels/HPF or 7 vessels/HPF dichomatized the tumors into two groups. Results were analyzed by chi-square testing.

${ }^{1}$ Department of Obstetrics \& Gynecology, ${ }^{2}$ Department of Pathology, ${ }^{3}$ Department of Biostatistics, ${ }^{4}$ Holden Comprehensive Cancer Center, The University of lowa, lowa City, lowa 52242

Corresponding author: Michael Goodheart, Department of Obstetrics and Gynecology, University of lowa, 3234 MERF, 200 Hawkins Drive, lowa City, IA, 52242. Telephone(319) 335-2015 michael-goodheart@uiowa.edu 
WESTERN ASSOCIATION OF GYNECOLOGIC ONCOLOGISTS (WAGO)

WAGO Annual Meeting

June 16-18, 2011

Stein Eriksen Lodge

Park City, Utah

\section{Results:}

\section{Conclusions:}

A total of 46 patients with primary Mutations of the p53 tumor suppressor epithelial ovarian cancer were included in our analysis. Serous histology was associated with absolute differences $(>7$ vessels/HPF) between MVD counts in primary vs. metastatic tumor sites $(p=0.07)$, as was an identified p53 mutation $(p=0.03)$. There was no association between absolute differences in MVD counts and grade $(p=0.30)$ or stage $(p=0.98)$. In our limited cohort there was no difference in survival between those with absolute MVD $>7$ vessels/HPF vs. $\leq 7$ vessels/HPF gene are associated with large differences ( $>7$ vessels/HPF) in MVD counts between primary and metastatic tumor sites in patients with epithelial ovarian cancer. These data are consistent with models demonstrating p53 mutation functions directly to influence angiogenesis. This information supports continued therapy and research involving angiogenesis inhibitors in patients with ovarian cancer, especially in the setting of increased differences in MVD between primary and metastatic sites.

P53, vascularity and ovarian carcinoma 